PART II

X-RAY ASTRONOMY 


\title{
PROGRESS IN THE OBSERVATIONAL X-RAY ASTRONOMY
}

\author{
MINORU ODA \\ Institute of Space and Aeronautical Science, University of Tokyo, Tokyo, Japan
}

\section{Introduction}

I shall review recent observational results on discrete $\mathrm{X}$-ray sources and on the diffuse background component. Thus far, approximately forty sources have been reported. It is to be noted that the sources can not be classified in only one or two categories and that there are a variety of the sources. Figure 1 shows the situation.

The majority of the sources apparently belonging to our Galaxy have not yet been identified with known astronomical objects. A part of these sources are unidentified probably because they have not yet been located with sufficient precision. Some sources, however, do not appear to generate detectable radio or optical emission at all in spite of careful search for the radio and optical counterparts.

Several sources have been identified with supernova remnants. In addition to the well studied Tau X-1 in the Crab Nebula, other supernova remnants, Cas A and the Tycho supernova, have been clearly shown to contain X-ray sources by the ASE* group (Gorenstein et al., 1970). Recently the LRL** group (Grader et al., 1970) and the ASE group (reported in this Symposium) indentified an extended soft and strong source with the Cygnus Loop. This supernova remnant has an age of a few $\times 10^{4} \mathrm{yr}$.

One or probably more extragalactic sources have been found; M87 has been well established and $3 \sigma \sim 4 \sigma$ and $3 \sigma$ of significance for the respective X-ray intensities of 3C 273 and Cen A were given by Bowyer et al. (1970).

Some sources including all of extragalactic sources appear to be synchrotron radiation (radio and/or light) emitters. On the other hand, there are sources which apparently do not emit synchrotron radiation.

\section{Sco X-1}

Various observational data have been accumulated for Sco X-1. Extensive optical observation of its time variations have been continued by Hiltner and Mook since 1967 (Hiltner and Mook, 1967). A regularity or a periodicity has been sought in the light curves without a positive conclusion.

The difficulty of finding a regularity comes from the fact that the optical data are a collection of short fragments of the light curve. The importance of acquiring long pieces of the light curve has been emphasized. Dr Hiltner organized a world-wide optical watch of Sco X-1 for this purpose for Apr. 23-May 16, 1970. So far as I understand simultaneous optical and X-ray observations were attempted with Vela satellites.

* American Science and Engineering, Inc.

** Lawrence Radiation Laboratory. 


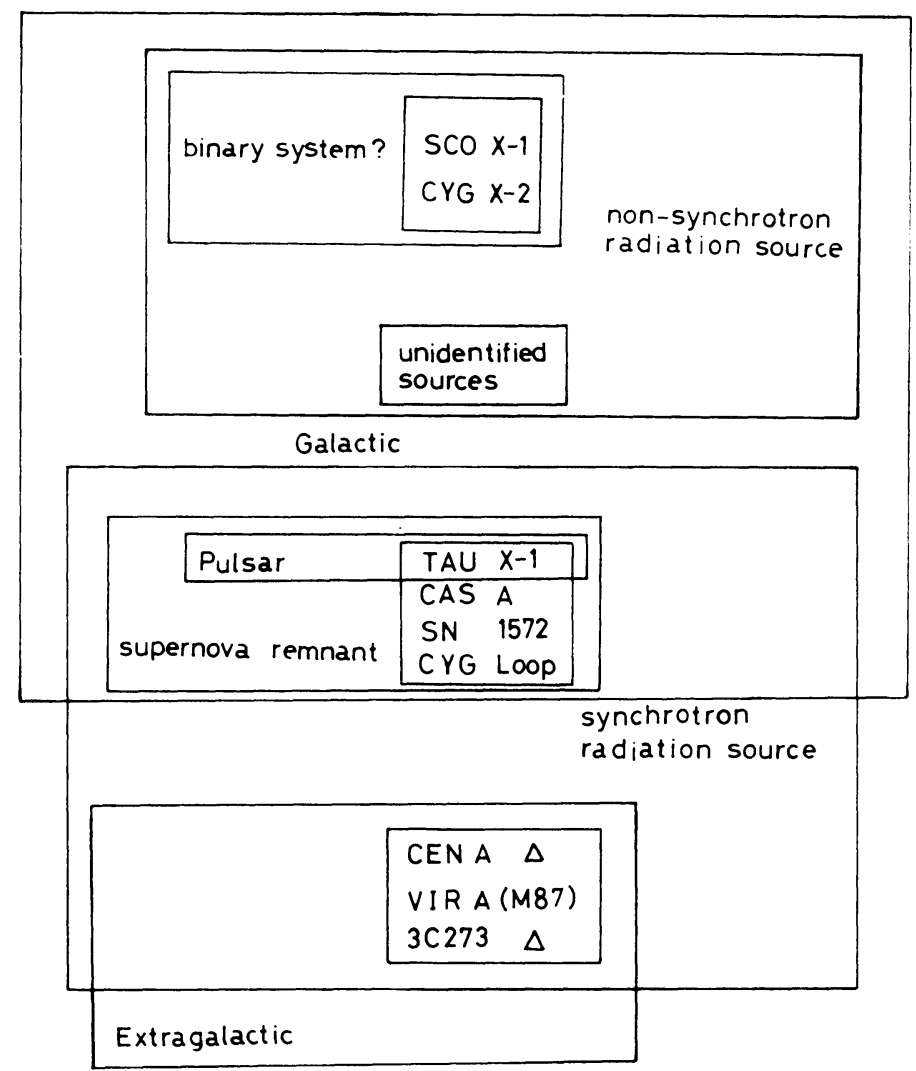

Fig. 1. Classifications of X-ray sources.

Two balloon X-ray observations were performed during this period by a collaboration of the Tata Institute group and the Japanese group, for which the results are not yet available.

The X-ray spectrum of Sco X-1, which has been well established up to now, is composed of an exponential part in the $1-10 \mathrm{keV}$ energy range and a flatter part beyond $30 \mathrm{keV}$ as indicated in Figure 2.

There is growing evidence for time variations of the X-ray spectrum. Figure 3 shows an example of the change of the spectrum in one day. It is a natural step in investigating the nature of Sco X-1 to study the time variation, in particular to study the correlation of the optical and the X-ray spectra by simultaneous observations.

Table I lists the rocket observations made with similar equipment and methods of analysis; the observations can be compared with each other.

If we assume a simple isothermal hot cloud model, we can then determine the parameters describing the cloud by these observations. Figure 4 shows the theoretical free-free emission spectrum which combines the X-ray and the optical spectra. The spectrum is exponential for $h v<k T$ and tends to be flat for $h v<k T$ with a slight 


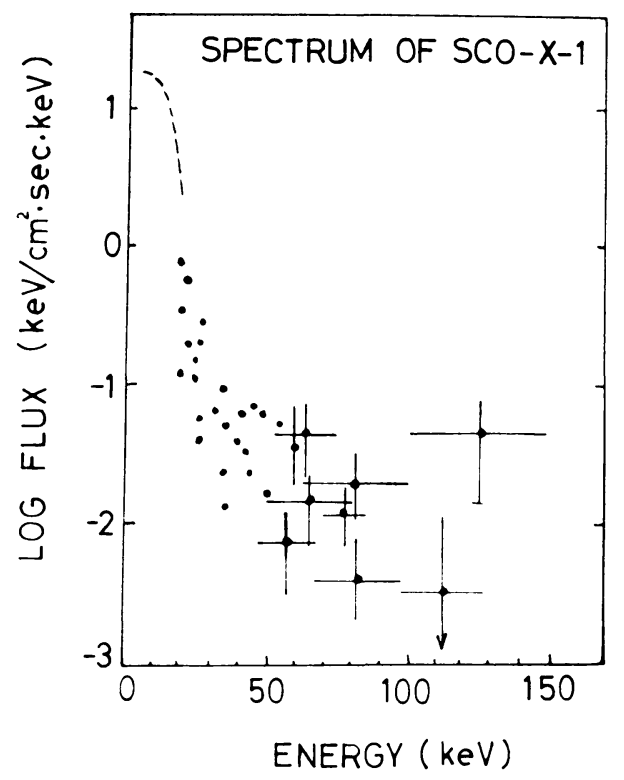

Fig. 2. Energy spectrum of SCO X-1. Data for the energy range $>20 \mathrm{keV}$ are presented by following balloon observations: Peterson et al. 18/6/1965, Boldt et al. 15/12/1966, Overbeck et al. 15/5, 24/5, 26/6/1967, Lewin et al 13/2/1967, Busselli et al. 29/2/1968, Sreekantan et al.

28/4, 22/12/1968, 16/4/1969.

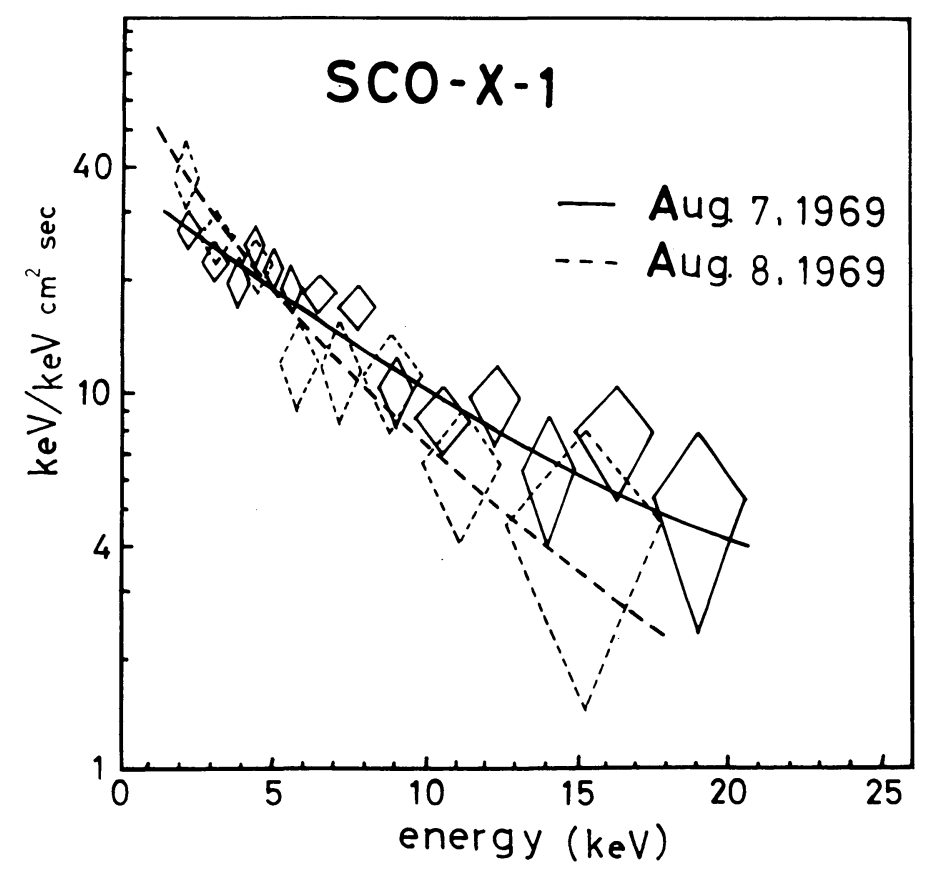

Fig. 3. Variation of Sco X-1 in one day. 
TABLE I

List of the rocket and optical observations of Sco X-1
Date

1967, May 18
1967, Sept. 29
1968, May 9
1968, May 19
1969, April 26
1969, April 28
1969, May 17
1969, May 24
1969, Aug 7
1969, Aug. 8

1967, May 18

1968, May 19

1969, April 26

1969, April 28

1969, May 17

1969, Aug 7

1969, Aug. 8

$\begin{array}{ll}\text { Effective } & n^{2} V \\ \text { temperature } & 4 \pi d^{2} \times 1015 \\ (k T \text { in keV }) & \left(\mathrm{cm}^{-5}\right)\end{array}$

$B$

$\left(\mathrm{cm}^{-5}\right)$

12.8

13.15

13.2

LRL

TERIS

TERLS

LRL

LRL

ISAS

ISAS

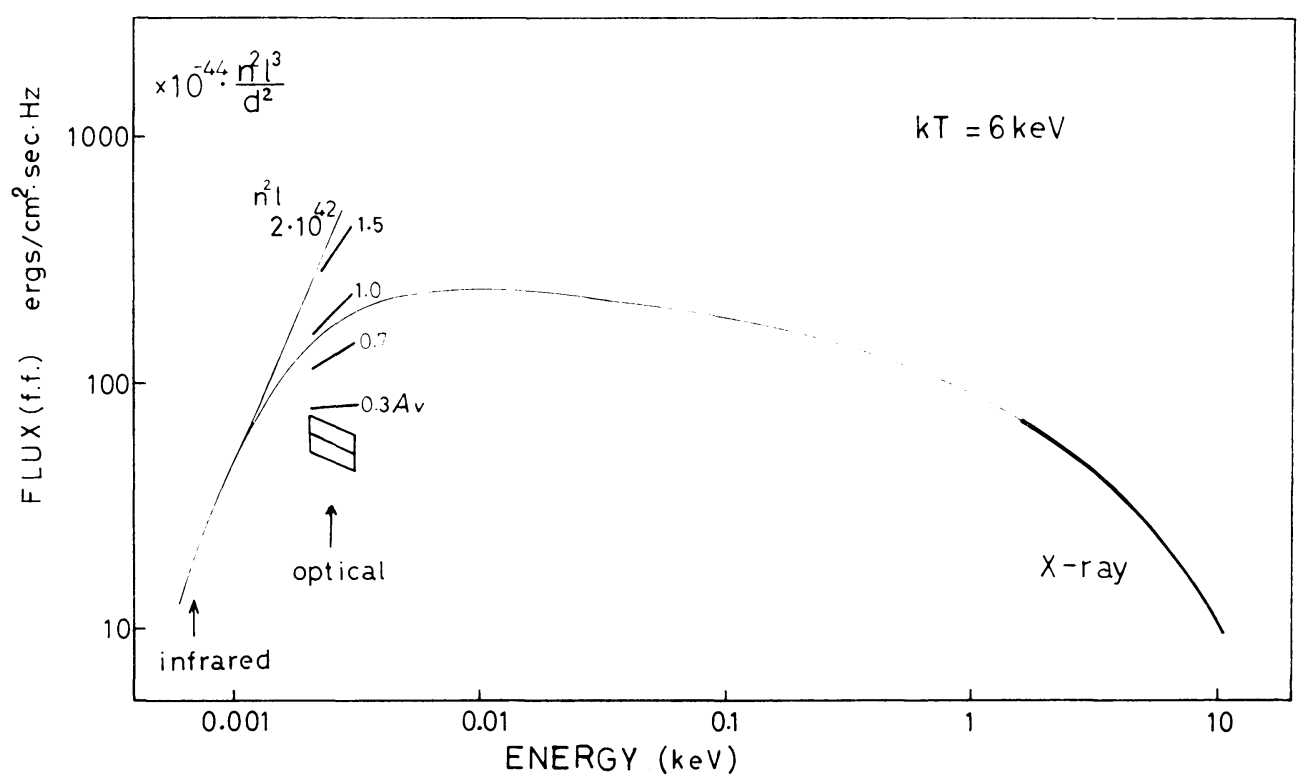

Fig. 4. Theoretical spectrum of an isothermal hot plasma.

increase at lower frequencies due to the Gaunt factor. For even lower frequencies where the source becomes opaque, the observed spectrum approaches the RayleighJeans spectrum after correction for interstellar extinction.

Comparison of the theoretical expression with the observed X-ray spectrum measures the parameters $T$ and $n^{2} r^{3} / d^{2}$, where $T$ is the temperature, $n$ and $r$ are the density and the size of the cloud respectively and $d$ is the distance to the source. The comparison of the optical spectrum with the theoretical expression determines $n^{2} r$ 
and $A$, where $A$ is the interstellar extinction. Figures 5 and 6 summarize the observational results for the relationships between $T$ and optical brightness, and $n^{2} r^{3} / d^{2}$ and $T$. The results are still premature, but they are consistent with the simple model described above; the dotted lines in the figures indicate the predicted relationships. From the

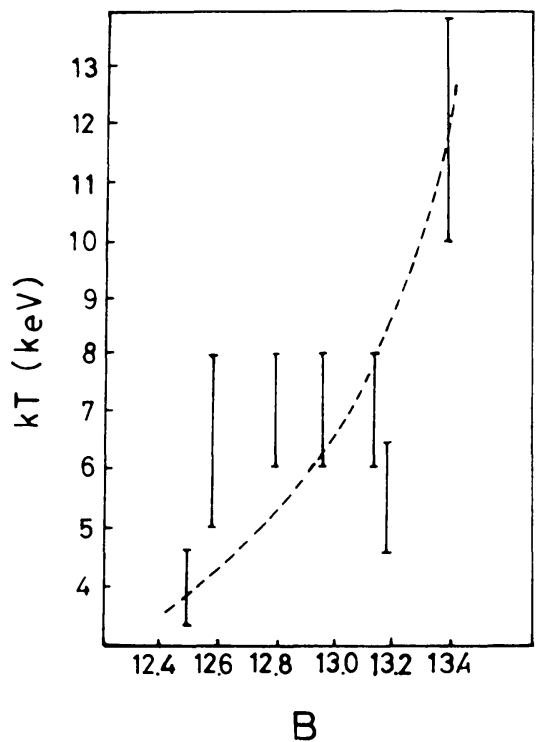

Fig. 5. Relationship between $T$, determined by X-ray observations, and optical (blue) brightness B.

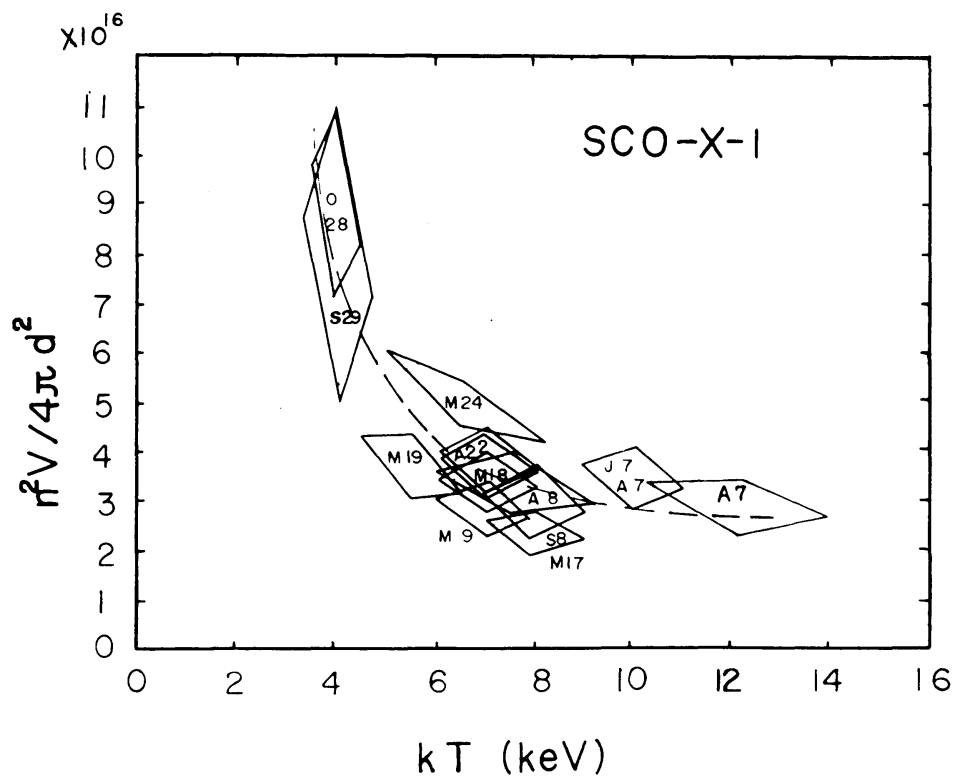

Fig. 6. Relationship between $n^{2-r^{3}} d^{*}$ and $T$. 
relationships we derive the combinations among $T, n^{2} r^{3} / d^{2}, n^{2} r$ and $A$ or, accordingly, $n, r$ and $T$ for an assigned $d$.

In the above argument the scattering of photons by electrons in the cloud was ignored. The analytical treatment of the problem is complicated. Loh and Garmire (1970) carried out Monte Carlo calculations for a number of combinations among the parameters. We have made an approximate expression for their calculations and applied it to wider combinations of the parameters. Table II shows the combinations of the parameters thus determined. Notice that the size of the cloud is equal to or

\section{TABLE II}

Relationships among $T, n$ and $r$ which describe the hot plasma model for assigned distances $d$. $t_{0}$ is the temperature of the cloud, while $T$ is the apparent temperature when the electron scattering is ignored.

\begin{tabular}{rlllllllll}
\multicolumn{3}{c}{$d=0.3 \mathrm{kpc}$} & \multicolumn{9}{c}{$d=1.0 \mathrm{kpc}$} \\
$k T(\mathrm{keV})$ & $A v(\mathrm{mag})$ & $k T_{0}(\mathrm{keV})$ & $r(\mathrm{~cm})$ & $n\left(\mathrm{~cm}^{-3}\right)$ & $\tau_{\mathrm{es}}$ & $k T_{0}(\mathrm{keV})$ & $r(\mathrm{~cm})$ & $n\left(\mathrm{~cm}^{-3}\right)$ & $\tau_{\mathrm{es}}$ \\
& & & & & & & & & \\
4 & 1.1 & 1.5 & $9.3 \times 10^{8}$ & $1.6 \times 10^{16}$ & 9.9 & 0.9 & $4.8 \times 10^{9}$ & $4.5 \times 10^{15}$ & 14.5 \\
6 & 0.9 & 2.7 & $5.6 \times 10^{8}$ & $2.4 \times 10^{16}$ & 9.1 & 1.5 & $3.0 \times 10^{9}$ & $6.3 \times 10^{15}$ & 12.9 \\
8 & 0.9 & 3.7 & $4.3 \times 10^{8}$ & $3.1 \times 10^{16}$ & 8.9 & 2.1 & $2.4 \times 10^{9}$ & $8.0 \times 10^{15}$ & 12.7 \\
10 & 0.9 & 4.4 & $3.8 \times 10^{8}$ & $3.7 \times 10^{16}$ & 9.4 & 2.5 & $2.1 \times 10^{9}$ & $9.7 \times 10^{15}$ & 13.5 \\
12 & 1.0 & 4.8 & $3.5 \times 10^{8}$ & $4.3 \times 10^{16}$ & 9.9 & 2.6 & $1.9 \times 10^{9}$ & $1.1 \times 10^{16}$ & 14.0
\end{tabular}

smaller than that of a white dwarf and that the relation between $T$ and $r$ is consistent with a gravitational confinement of a hot plasma by a central body with a fraction of solar mass. It is a tempting idea to consider a hot plasma cloud which is confined around a neutron star by its gravitational force; the plasma being continuously supplied from outside and being heated by the intense magnetic field of the spinning neutron star.

The importance of finding line spectra as a crucial test for the hot cloud model should be stressed. An $\mathrm{Fe}$ line has been reported with $3.3 \sigma$ of significance, but it is not yet very convincing (Holt et al., 1969).

Another remarkable feature of Sco X-1 is its flaring. Flare X-rays observed from balloons have been reported by the MIT and Tata groups (Lewin et al., 1968; Agrawal et al., 1969) and a number of X-ray flares have been observed by the UCSD* group with OSO 3 (Hudson et al., 1970). Figure 7 shows the latest observation of a flare or a large fluctuation by the MIT group (Lewin, 1970). A simultaneous X-ray and optical observation was done on the occasion of an optical flare by the UCSD group, and a positive correlation was found (Hudson et al., 1970). More such observations obviously will be valuable.

* University of California, San Diego. 


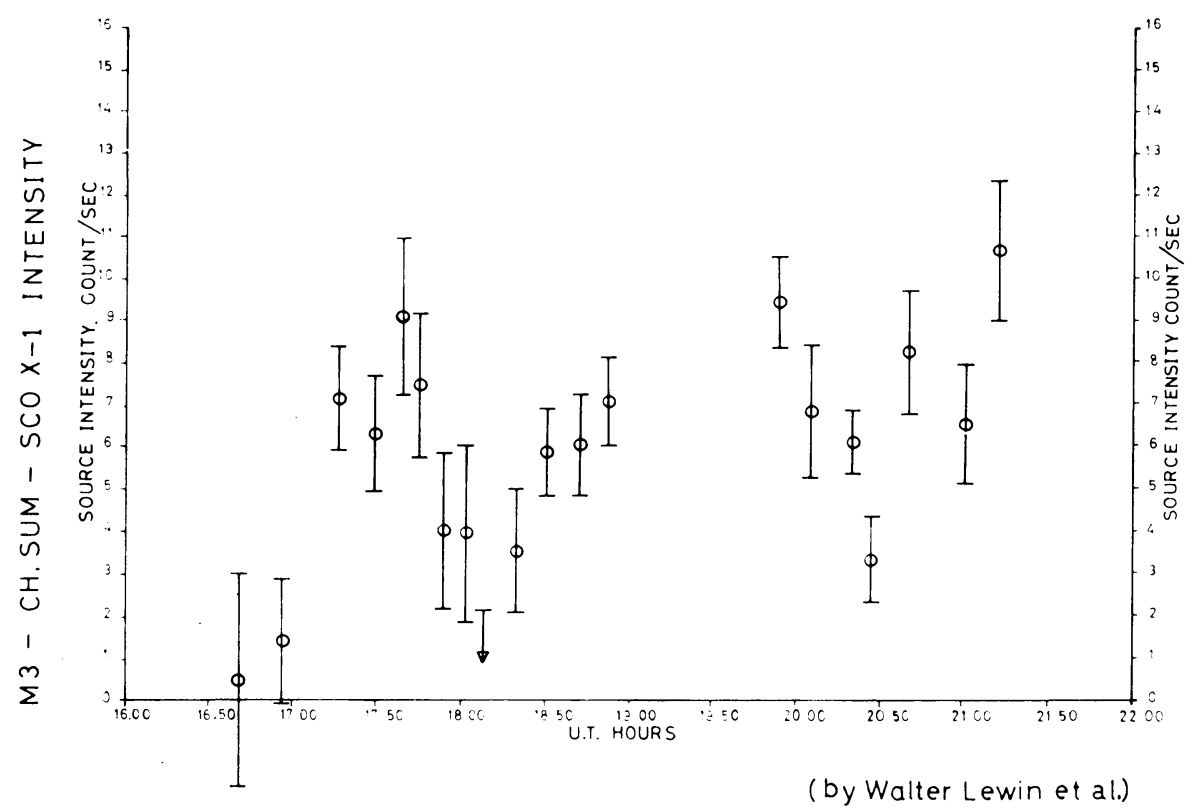

Fig. 7. A large fluctuation of Sco X-1. (MIT balloon observation by courtesy of W. Lewin.)

\section{Nova-like X-Ray Source}

At the end of July 1969 Conner et.al. announced the appearance of a strong new source observed with the Vela satellites. Figure 8 shows the time profile of the source, which first appeared between July 6 and July 9. The intensity remained nearly constant for about two months; the object became dimmer gradually starting in early September and disappeared by the end of September (Evans et al., 1970).

Two rocket observations and two balloon observations were performed during this period (Kitamura et al., 1969; Fujii et al., 1969). Figure 9 represents the spectrum of the source. We need strong absorption below $3 \mathrm{keV}$ if we apply a hot cloud model. The columnar density of interstellar gas corresponding to this absorption is estimated as $3 \times 10^{22}$ atoms $/ \mathrm{cm}^{2}$.

In view of the high galactic latitude of the source $\left(25^{\circ} \mathrm{N}\right)$ where the density is at most $1 \times 10^{21}$ atoms $/ \mathrm{cm}^{2}$, a dense circumstellar cloud must be considered. This conclusion may explain the fact that an immediate survey of the sky area of the new source by optical astronomers had not yielded any positive results. (Private information by W. Hiltner.)

This nova-like behavior reminds us of Cen XR-2 which appeared 1966-67. Figure 10 summarizes the observational results on both sources. In spite of the apparent similarity there appear to be some definite differences.

(1) Strong absorption, probably due to a dense circumstellar gas, is concluded only for GX $333+25$. 


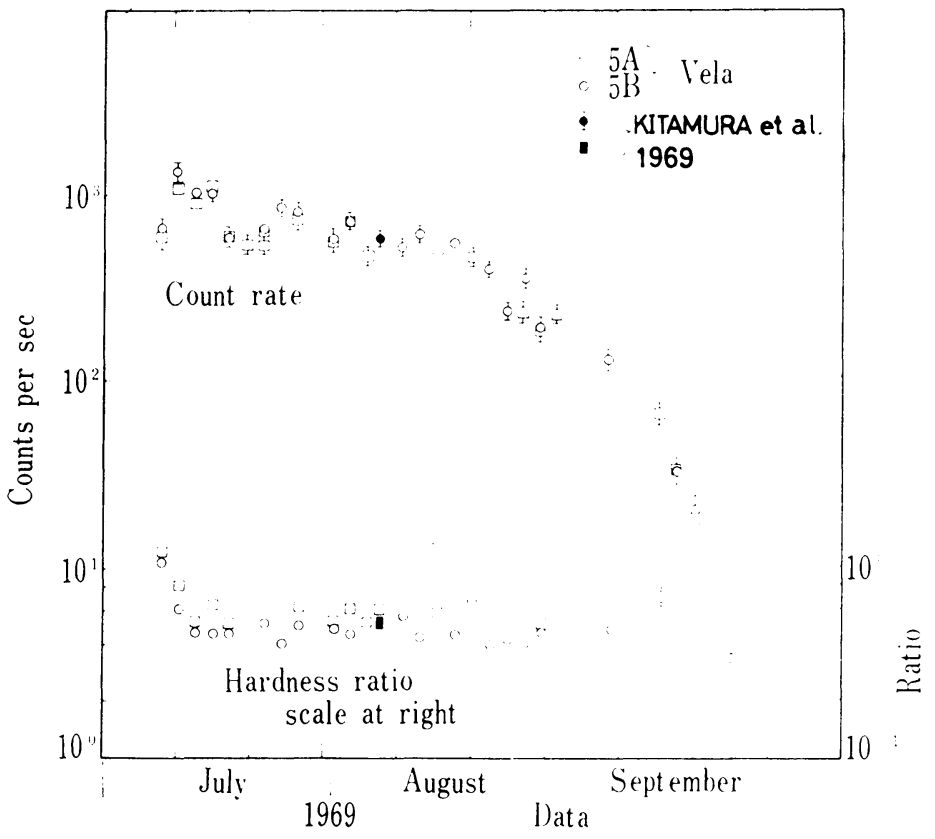

Fig. 8. Time history of a nova-like X-ray source.

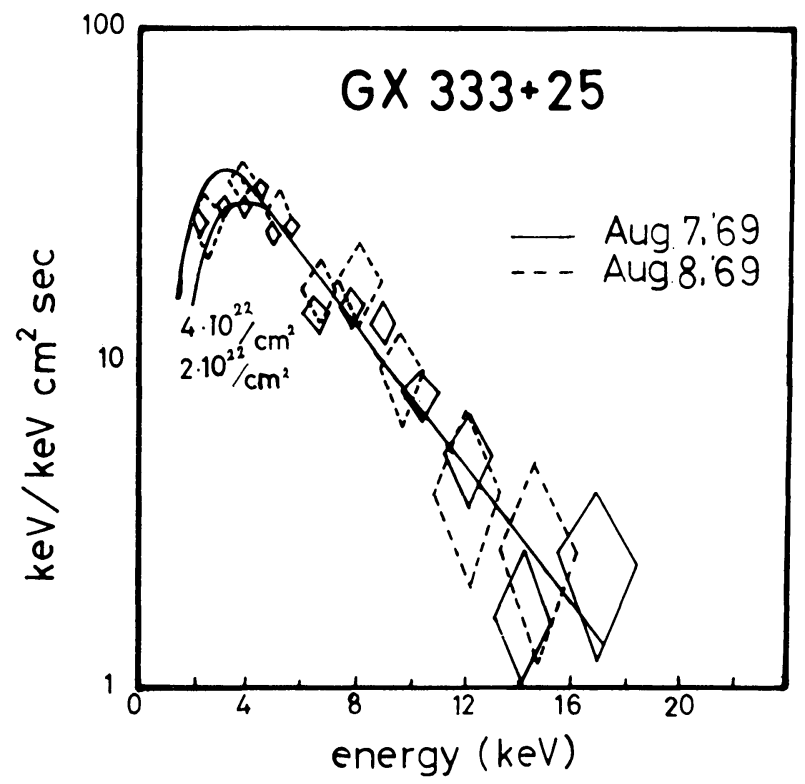

Fig. 9. The energy spectrum of the source. 
(2) Variation of the spectrum (softening) was observed for Cen XR-2, but the hardness of the spectrum of GX $333+25$ remained constant while the source had faded.

(3) After Cen XR-2 disappeared in 2-10 keV range, its reappearance was observed by a balloon experiment.

There are indications of the sudden appearance and disappearance of weaker sources. Such temporal behavior of an X-ray source may be a common phenomenon and watching for X-ray novae will undoubtedly be important.

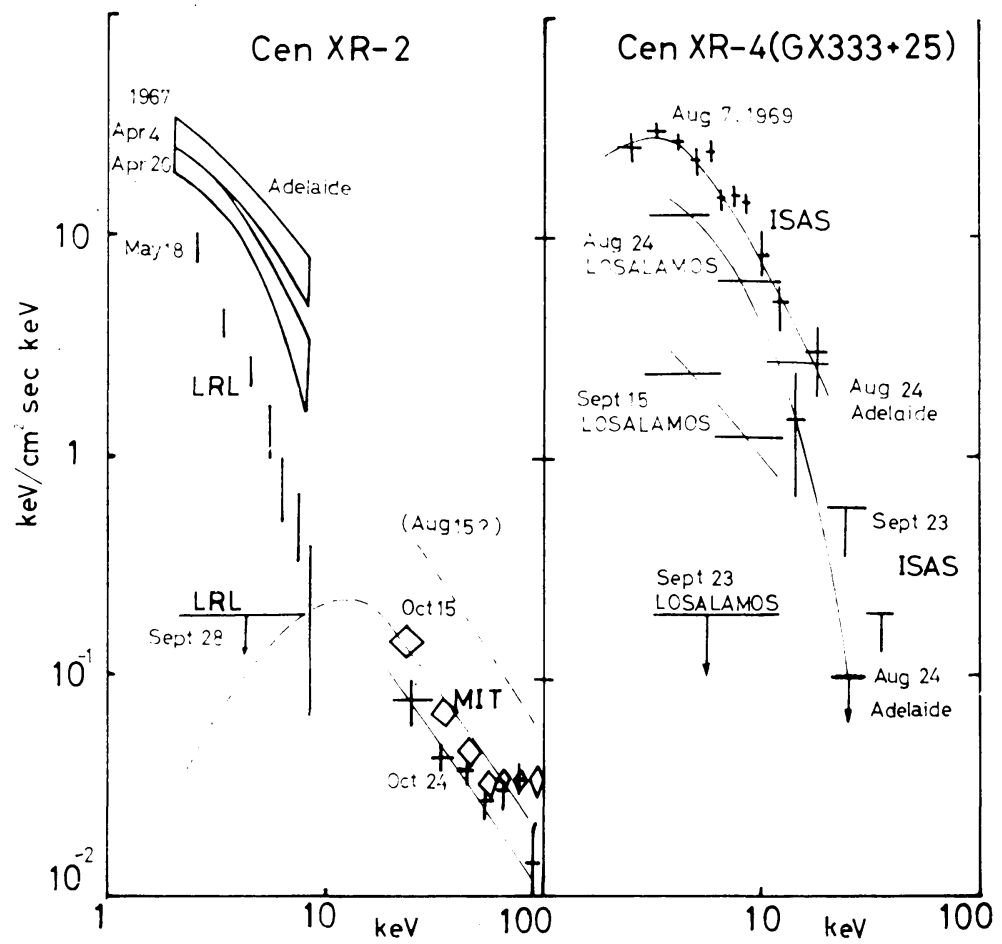

Fig. 10. Comparison of GX $333+25$ with Cen XR-2.

\section{Tau X-1}

The power law spectrum has been well established up to $100 \mathrm{keV}$. The existence of an X-ray pulsar has been clearly established since the Rome IAU Symposium (1969) by the groups at NRL, MIT, Goddard, Rice University and the Tata Institute (Fritz et al., 1969; Bradt et al., 1969; Boldt et al., 1969; Fishman et al., 1969).

Its relative intensity to the stable component that is the diffuse source appears to increase with increasing energy as shown in Figure 11. Latest observations of the $\mathrm{X}$-ray pulsar by the Wisconsin group are shown in Figure 12. Notice that the second peak is harder than the first. 


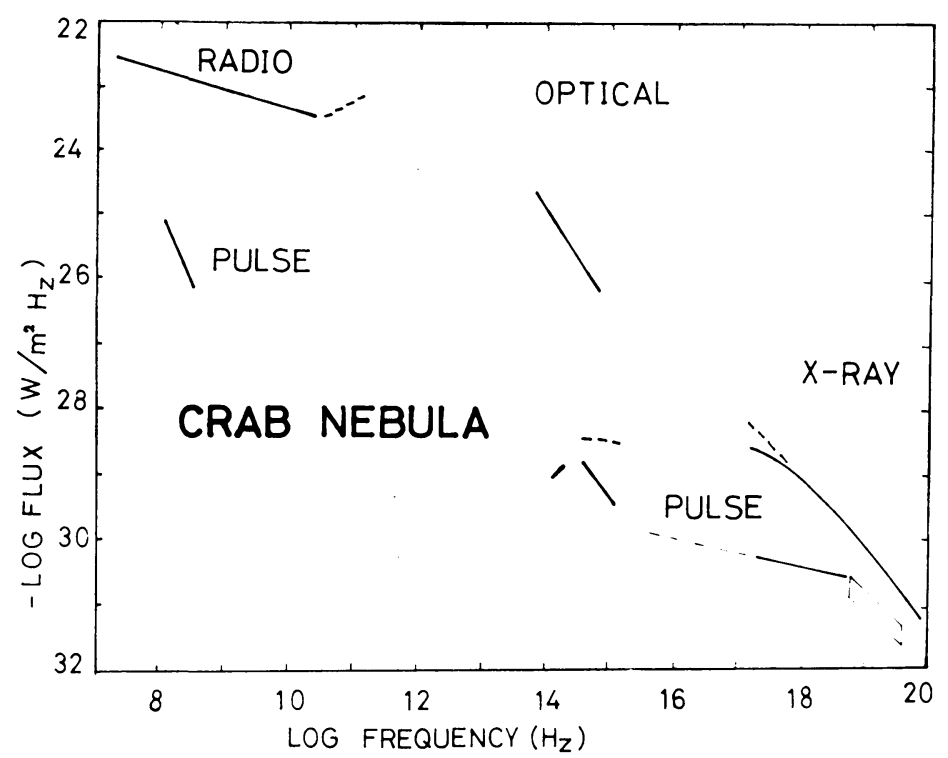

Fig. 11. Energy spectra of the stable and the pulsar component of the Crab Nebula.

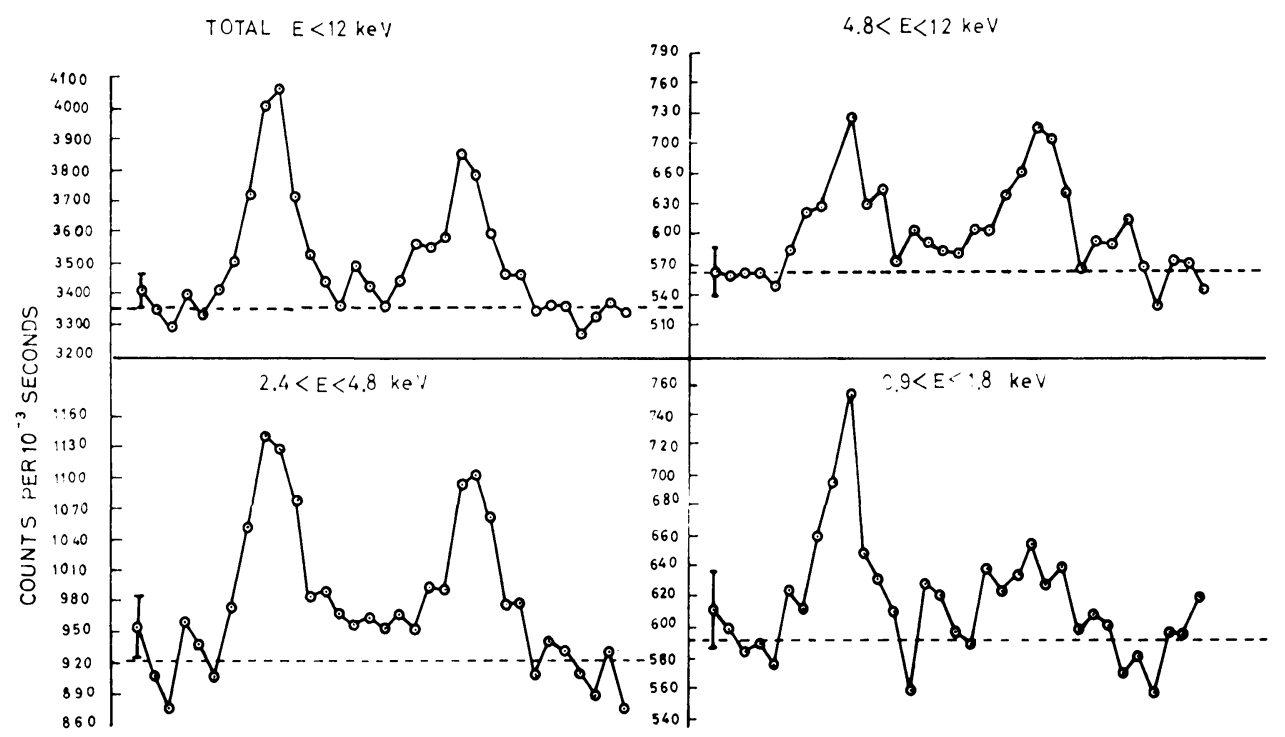

Fig. 12. X-ray pulsar in the Crab Nebula (Wisconsin group).

\section{Soft X-Ray Sources}

A few soft X-ray sources have been reported. The LRL group found Vel XR-2 to be visible only below $0.5 \mathrm{keV}$ (Grader et al., 1970). The Wisconsin group reported GX 341-6 (Sco XR-3) in 1.5-6.6 keV band. Estimated temperatures for both sources were as low as $<2.10^{7} \mathrm{~K}$. The Cygnus Loop appears to be soft. its temperature being 
$6 \times 10^{6} \mathrm{~K}$. Thus far the number of soft sources is not sufficient to conclude the temperature distribution of X-ray sources by means of a statistical argument taking into consideration the interstellar extinction of the soft sources. The importance of surveying more soft sources is to be stressed.

\section{Identifications of X-Ray Sources}

Efforts of optical and radio identifications of X-ray sources have been continued. The identifications of several sources near the Galactic center whose precise locations have been determined by MIT experiments with the rotating modulation collimator

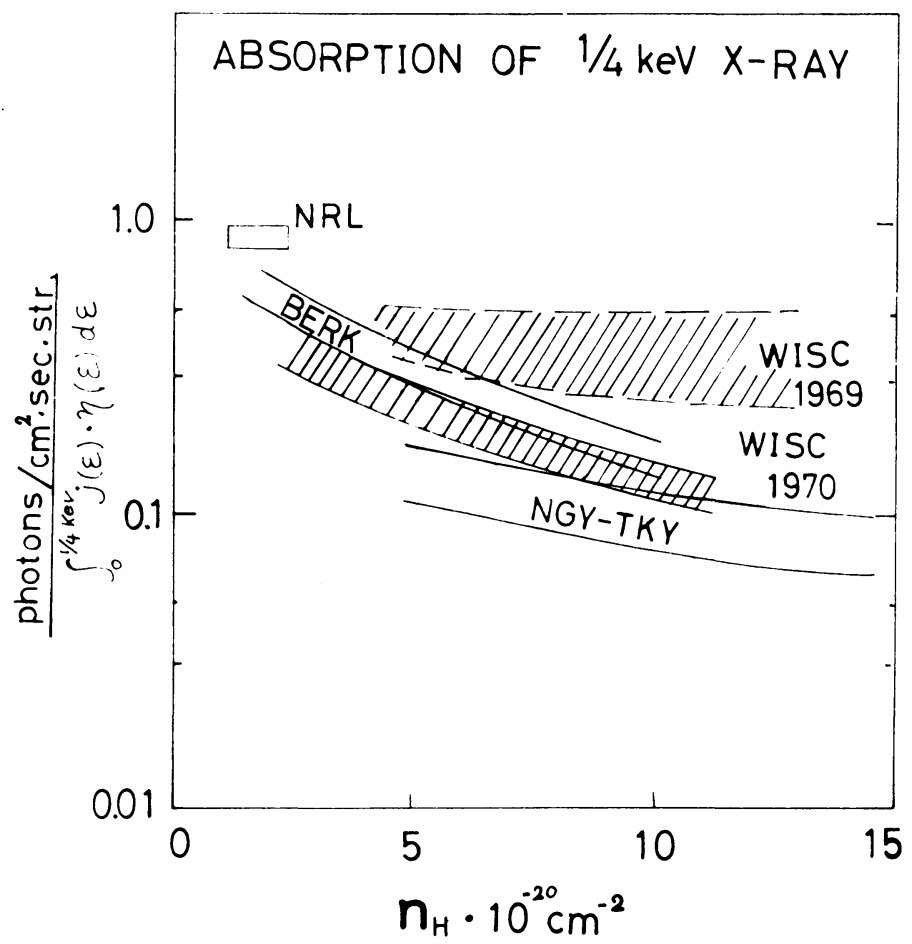

Fig. 13. Interstellar attenuation of the $\frac{1}{4} \mathrm{keV}$ X-rays. BERK: Berkeley group, WISC: Wisconsin group, NGY-TKY: Nagoya-Tokyo group, $j$ and $\eta$ are the spectrum and the detection efficiency for energy $\varepsilon$ respectively.

on board an aspect-controlled rocket, thus far, have been unsuccessful (Schnopper et al., 1970; Kunkel et al., 1970; Andrew et al., 1970). It has been accordingly concluded that these sources are not recent supernova remnants.

The location of Cyg X-1 determined by the ASE group (Giacconi et al., 1967), the location given by a balloon observation with a modulation collimator (Matsuoka et al., 1970), and the location of a strange blue star in this general area found by 
Osawa of Tokyo Observatory (Ishida et al., 1969) are all contradictory with each other by a fraction of a degree.

\section{Diffuse Background}

Extensive scan of the entire sky by OSO 3 (UCSD group) (Schwartz et al., 1970) in $7.7 \sim 38 \mathrm{keV}$ range confirmed a $10 \%$ increase of X-ray intensity at the Galactic plane that is consistent with the existence of a hump at the Galactic plane reported by the Leicester group a year ago (Cooke et al., 1969). Otherwise the entire sky is smooth, the upper limit on the intensity inhomogeneities of the about $12 \%$ radius being $3 \%$ and that of the smooth variation in the entire sky being $1 \%$.

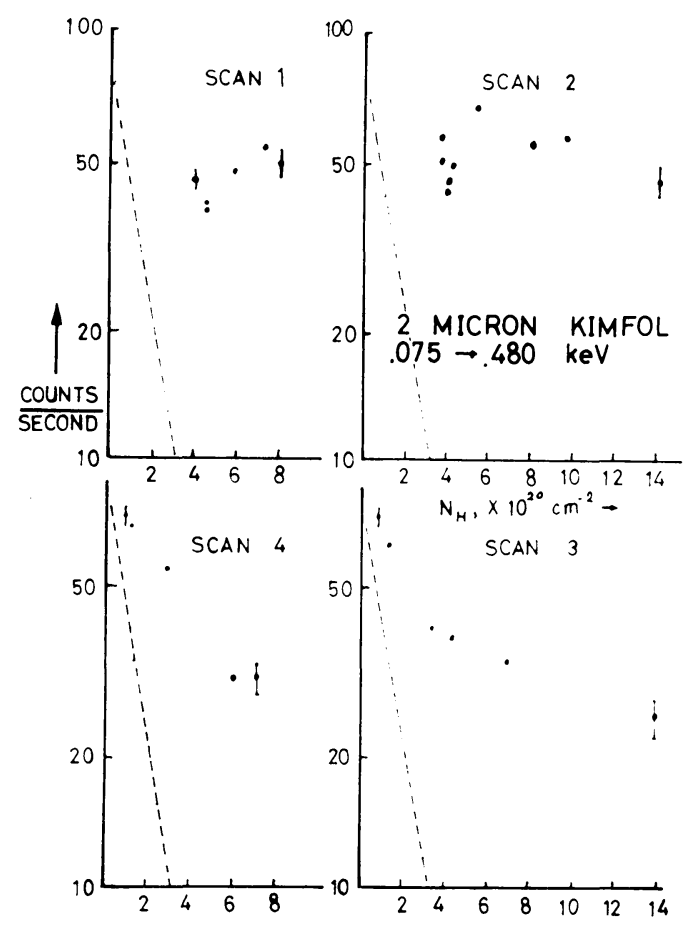

Fig. 14. Wisconsin observation of the very soft diffuse background for different scan paths in the Galactic coordinates (by the Wisconsin group).

The general uniformity of X-ray intensity above several keV suggests an extragalactic origin for a large fraction of the X-rays. The increase of very soft X-rays $(<1 \mathrm{keV})$ with increasing galactic latitude (decreasing columnar density of the interstellar gas) was considered to support its extragalactic origin.

However, the details of the results on very soft X-rays appear more complicated than was expected in terms of the interstellar absorption of extragalactic X-rays. One 
year ago, when we tried to compile the results of $\frac{1}{4} \mathrm{keV}$ observations, we met considerable difficulties though a general trend of interstellar absorption was clear. Figure 13 summarizes the results of the various group (Oda, 1970). These results were not precisely consistent with each other, and the nature of the attenuation was not as expected. These early results were interpreted in terms of the clumpiness of interstellar gas clouds (Bowyer and Field, 1969; Bunner et al., 1969) or local sources of X-rays.

Figure 14 shows the recent observation by Wisconsin group which clearly shows the

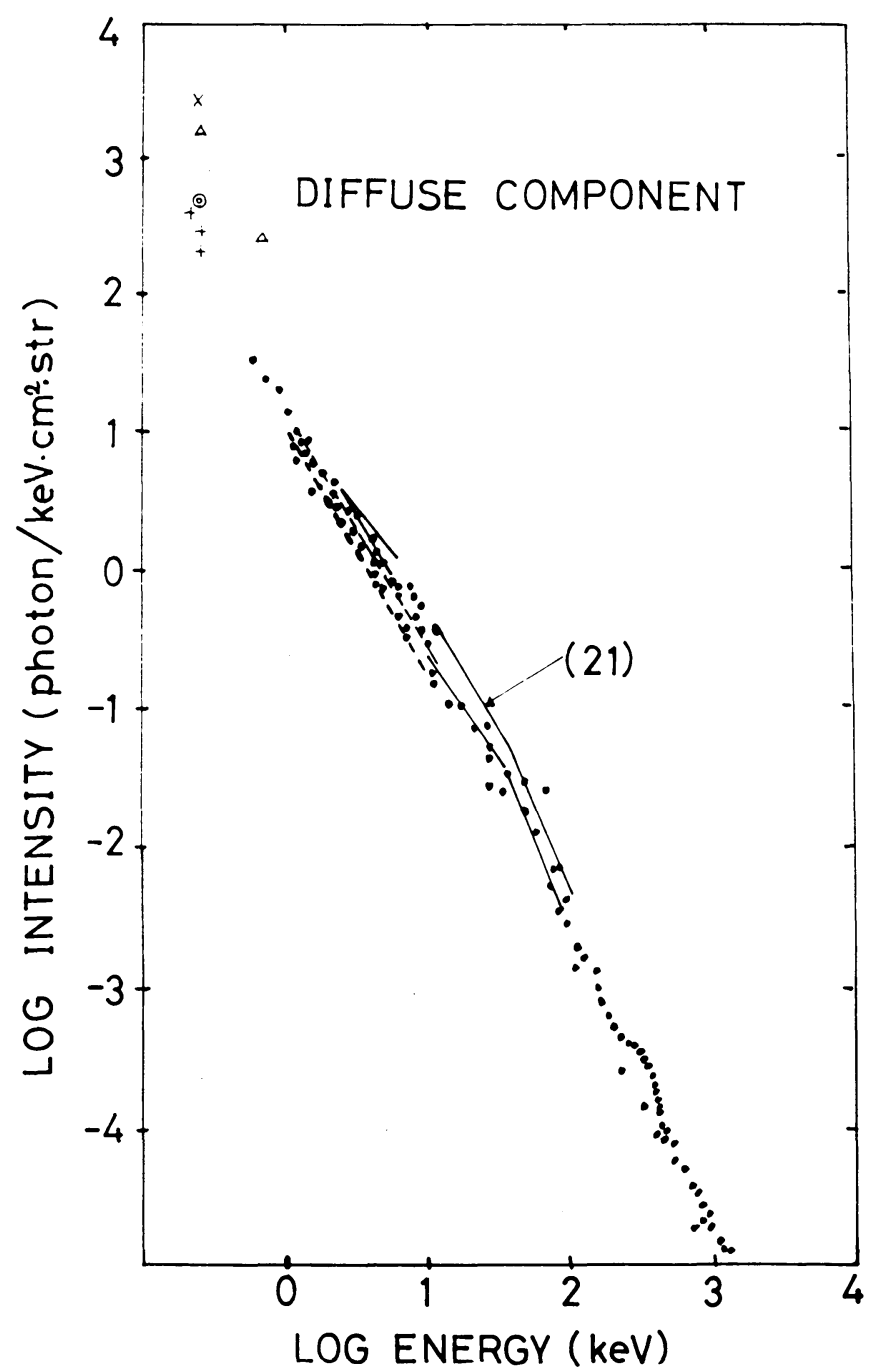

Fig. 15. Energy spectrum of the diffuse component. Rocket, balloon and satellite data are summarized. The double circle shows the compiled result from measurements of $\frac{1}{4} \mathrm{keV}$ by various groups corrected for the interstellar attenuation. 
irregular nature of the absorption due to the differences in the nature of the absorption at different galactic longitudes.

Figure 15 shows the latest version of the energy spectrum including the results by OSO 3. A break in the spectrum at $\sim 40 \mathrm{keV}$ that has been suspected earlier has now become more convincing.

\section{Acknowledgements}

I wish to acknowledge contributions of those who made their latest results available before publications.

\section{References}

Agrawal, P. C., Biswas, S., Gokhale, G. S., Iyengar, V. S., Kunte, P. K., Manchanda, R. K., and Sreekantan, B. V.: 1969, Nature 224, 51.

Andrew, B. H., Purton, C. R., Rappaport, S., Bradt, H., and Schnopper, W. W.: 1970 (preprint).

Boldt, E. A., Pesai, U. D., Holt, S. S., Serlemitsos, P. J., and Sirerberg, R. F.: 1969, Nature 223. 280.

Bowyer, C. and Field, G.: 1969, Nature 223, 573.

Bowyer, C. S., Lampton, M., and Mack, J.: 1970, Astrophys. J. 161, L1.

Bradt, H., Rappaport, S., Mayer, W., Nather, R. E., Warner, B., Macfarlane, M., and Kristian, J.: 1969, Nature 222, 728.

Bunner, A., Coleman, P., Kraushaar, W., McCammon, Dr., Palmieri, T., Shilepsky, A., and Ulmer, M.: 1969, Nature 223, 1222.

Cooke, B. A., Griffiths, R. E., and Pounds, K. A.: 1969, Nature 224, 134.

Evans, W. D., Belian, R. D., and Conner, J. P.: 1970, Astrophys. J. 159, L57.

Fishman, G. J., Harnden, F. R., and Haymes, R. C.: 1969, Astrophys. J. 156, L107.

Fritz, G., Henry, R. C., Meekings, J. F., Chubb, T. A., and Friedman, H.: 1969, Science 164, 709.

Fujii, M., Matsuoka, M., Miyamoto, S., Nishimura, J., Oda, M., Ogawara, Y., Ohta, S., and Takagishi, K.: 1969, Proc. of ISAS Balloon Symposium, 247.

Giacconi, R. P., Gorenstein, P., Gursky, H., and Waters, J. R.: 1967, Astrophys. J. 148, L119.

Gorenstein, P., Kellogg, E. M., and Gursky, Y.: 1970, Astrophys. J. 160, 199.

Grader, R. J., Hill, R. W., Seward, F. D., and Hiltner, W. A.: 1970, Astrophys. J. 159, 201.

Grader, R. J., Hill, R. W., and Stoering, J. P.: 1970, Astrophys. J. 161, L45.

Hiltner, W. A. and Mook, D. E.: 1967, Astrophys. J. 150, L23, 851 and private communications.

Holt, S. S., Boldt, E. A., and Serlemitsos, P. J.: 1969, Astrophys. J. 158, L155.

Hudson, H. S., Peterson, L. E., and Schwartz, D. A.: 1970, Astrophys. J. 159, L51.

Ishida, G., Kondo, M., Nishimura, S., Osawa, K., and Ichimura, K.: 1969, Tokyo Astron. Bull. 2nd, Series No. 196, 2277.

Kitamura, T., Matsuoka, M., Miyamoto, S., Nakagawa, M., Oda, M., Ogawara, Y., and Takagishi, K: 1969, Nature 224, 784.

Kunkel, W., Osmer, P., Smith, M., Hoag, A., Schroeder, D., Hiltner, W. A., Bradt, H., Rappaport S., and Schnopper, H. W.: 1970 (preprint).

Lewin, W. H. G.: 1970 (private communication).

Lewin, W. H. G., Clark, G. W., and Smith, W. B.: 1968, Astrophys. J. 152, L55.

Loh, E. and Garmire, G.: 1970 (private communication).

Matsuoka, M., Miyamoto, S., Nishimura, J., Oda, M., Ogawara, Y., and Wada, M.: 1970, in L. Gratton (ed.) 'Non-Solar X-and Gamma-Ray Astronomy', IAU Symp, 37, 130.

Oda, M.: 1970, in L. Gratton (ed.), 'Non-Solar X- and Gamma-Ray Astronomy', IAU Symp. 37, 260.

Schnopper, H. W., Bradt, H. V., Rappaport, S., Boughan, E., Burnett, B., Doxsey, R., Mayer, W., and Watt, S.: 1970 (preprint).

Schwartz, D. A., Hudson, H. S., and Peterson, L. E.: 1970 (private communication). 


\section{DISCUSSION}

L. W. Acton: Do you feel that there is evidence for a secular (long term) variation of Sco X-1?

$M$. Oda: No, I do not think we have any positive evidence for a secular variation.

$E$. B. Jenkins: What average space density and galactic scale height do you assume in your derivation of column densities in your illustrations of the diffuse soft X-ray attenuation?

M. Oda: I used the compiled results by Kraushaar and Garmire in Space Science Reviews. The latest compilation by Daltabuit is also useful.

H. F. Helmken: What type of model for Sco X-1 will include the observed flattening of the X-ray spectrum around 50 to $100 \mathrm{keV}$ ? Does it imply a non-thermal component?

M. Oda: It is possible that the spectrum of Sco X-1 is composed of a thermal and a non-thermal component and that the X-ray spectrum of 2 to $20 \mathrm{keV}$ is the former and the latter appears in the radio range and the hard $\mathrm{X}$-ray range.

J. E. Grindlay: Do you think that the observed apparent inverse relation between angular size and effective temperature of the X-ray source Sco X-1 lends any support to the hypothesis that the source may be a black hole or neutron star accreting mass from a interstellar cloud?

M. Oda: Yes, I think so. Besides, the radius and the temperature of the cloud are consistent with a model of the gravitational confinement of the hot plasma around a neutron star with a fraction of the solar mass. 